\title{
Strength and Weakness Characteristics of Using Polymer Based Alternative to Steel Mesh In Underground Coal Mine Strata
}

\author{
Akash Talapatra ${ }^{1 *}$ \\ ${ }^{1}$ Department of Petroleum \& Mining Engineering, Cuet,Chittagong, Bangladesh \\ *Corresponding author: talapatraakash@gmail.com
}

(Received: June 15 ${ }^{\text {th }}, 2019$; Accepted: July 1 ${ }^{\text {st }}$, 2019)

\begin{abstract}
Nowadays a viable development of polymer based material as an alternative to the steel mesh material has been increased at a notable amount for providing enough support in the underground roadways. This feasibility study done on the development of using polymer based alternative is related with both of the chemical \& physical properties of the selected material. If the polymer alternative has a considerable amount of capability to tolerate all the physical \& material constraints equal to or above the normal steel mesh, then the possibility of using polymeric alternatives have been increased at a greater extent. For this reinforcement test, an experiment practice would be done by comparing the mechanical properties of the steel mesh with the polymeric material such as modulus property, elongation-at-break, yield stress etc. After that, a suitable polymer based alternative will be applied for underground roadway support in upcoming days.
\end{abstract}

Keywords: Cure Characteristics; Flexural Strength; Maximum Deflection; Polymeric Alternative; Skin Confinement

\section{Introduction}

Steel mesh is generally used as a structural material made of both with iron \& carbon (approximate amount of carbon 0.2 to 1.5 ). The steel mesh is very hard, strong $\&$ has low brittle index. For that, the use of steel mesh has been increased in coal mine working space for constituting mine roadways. There are some underground coal mines existing in the earth where there is a persistence of having faulty ground condition \& that coal mine locations may also have the possibility of occurring ground vibration. As a result, the ground strata or layers can be fractured accidently along with an undesirable coal fragmentation. In this case, the steel mesh can act as the supporting role to provide the necessary confinement to the coal mine strata so that the coal seam cannot be fallen from the roof \& cage due to weak reinforcement of mine bolts.

Here the steel mesh is installed in mine roadway for reinforcing the development in a totally manual process that is considered as both slow \& risky steps. A common technology known as self-drilling bolting technique that is fully developed with potential automation has been investigated broadly over a few years. This technique is almost functioned with manual processes. But nowadays, there is a process to find out an alternative of the steel mesh that can be installed totally in automatic way $\&$ can provide the proper automation to the mine roadways along with the development of the self-drilling bolting technique. The coal mining companies in collaboration with some technical researchers have been incorporated to this research to find out the appropriate alternatives to steel mesh, which must have the following requirements: 
- Having the same or higher level of effective skin confinement to that steel mesh

- Requiring the least amount of human intervening action for the installation of it

- Having the capability of removing the personnel from the working face area

- Enabling the development rate of underground mine roadway construction

- Providing the adequate safety for using \& handling

- Having the maximum cost effectiveness

\subsection{Thin Spray-On Liners}

Thin spray-on liners also known as 'TSLs' are basically the materials produced from polymer compounds used as a supporting alternative to the steel mesh for the underground mine roadways. They are designated such a way that they can provide an additional supporting system to the steel mesh. Such a type of polymer based material is poly-urea (also known as crosslinking polyurethane in the market) which is produced from a mixture of approximately 20 available market products. The other mostly used polymer based material is cement reinforced material included with a water dispersible system produced from the ethylene-vinyl acetate copolymer (Espley \& S.J 1999, Potvin et al. 2004). The applicability of both of these materials is generally restricted in some points for having of the relatively limited range properties of the polymeric materials. Not only that, but also the flexibility of this polymer based material is low as there is a formation of some cementitious materials though they have higher strengths.

\subsection{Underground Prevailing Condition}

The existing conditions of underground are not same in every mine. The applications of roadway rib facilitation can extend from development of a single rib bolt to three or more numbers of rib bolts in per mesh number $\&$ finish roofing to floor meshing that is dependent on the rate of surface movement $\&$ the experienced soundness of the structural coal ribs. The type of mesh used in this coal rib roof generally in full width \& has a continuous type of bolting in the square pattern. The range of exercising the roadway development is started from 'cut-\&-flit' application to the bolting application depending on the durability of the coal strata. Some types of issues found in some underground mines are water \& gas drainage problems along with the variation of $\mathrm{pH}$ levels. Therefore, the materials selected for applying in these conditions must have the appropriate level of support for the long duration. The main purposes of this paper are discussing some issues related with the installation of new polymer based material that can replace the steel mesh from supporting the coalmine strata $\&$ employing some strategies to solve these issues.

\section{Polymeric Skin Confinement Property Requirements}

The expectable properties of material in a polymeric skin confinement system follow:

- Without any type of slumping, the material should have the ability to spray in the system;

- The system cannot omit any type of irritant or toxic gas during the application time;

- Should be set up initially in the full strength for curing;

- Not only that, the system should also have the full strength development from minutes to hours;

- Better adhesion power to coal or rock roof than steel for full curing;

- Sensitive to coal or rock dust \& water \& not sensitive to $\mathrm{pH}$ variation;

- Better flexibility with an enough rupture strength;

- Able to seize the roof \& rib spalls;

- Capacity of piling increased by buttressing fillers;

- Fire \& explosion prevention. 


\subsection{Material Section Process}

The required physical \& chemical properties of materials have been investigated to find out a number of acute polymeric alternatives. A summarization of the material selection process has been shown in Figure 1 by designing a flow chart. This process is controlled with a "yes-no" criteria \& formed with four important following stages:

- Cure characteristics;

- Flexural characteristics;

- Rheological characteristics;

- Environmental criteria.

\subsection{Cure Characteristics}

The theoretical dilution of events is set up to cut the driving roadway by implying a successive mining machine along with employing a new confine measurement to drill \& place bolts. In order to accomplish this process in the least duration, a confined polymeric substance requires to forwarding from liquid to solid that may take a few minutes after a spray action is referred as a curing process.

The governing chemical process in curing tends to increase the speed of switching of scattered liquid to a solid polymer. There is a number of cure chemistries which are needed for rapid curing of the solid polymer by only the emitted water without any type of molecule discharge.

One of the necessary chemical curing of the process is the polymer crosslinking that is performed in two stages associated with gelation \& vitrification processes (Chang \& Chen 1987, Martin et al. 2000, Cook et al. 2001). Cure monitoring can be designed with managing a differential scanning calorimeter (DSC) that is used for measuring the heat flux by considering both time \& temperature as function. Figure 2 has shown a basic representation of DSC measurement. During the induction period, there is no heat flux occurred, can subsist from seconds to hours based on the types of accelerators \& promoters used. In this first stage, the substance can build a gel along with occurrence of an exotherm process that is known as a gelation process. At this stage, the material or object is dimensionally durable though it is not in structure \& then the object is drilled \& strongly bolted. Finally, in the second stage, the vitrification process occurs also subsists from seconds to hours like the previous stage. In this stage, the material or object forms a glass \& acquires the high structure strength. 


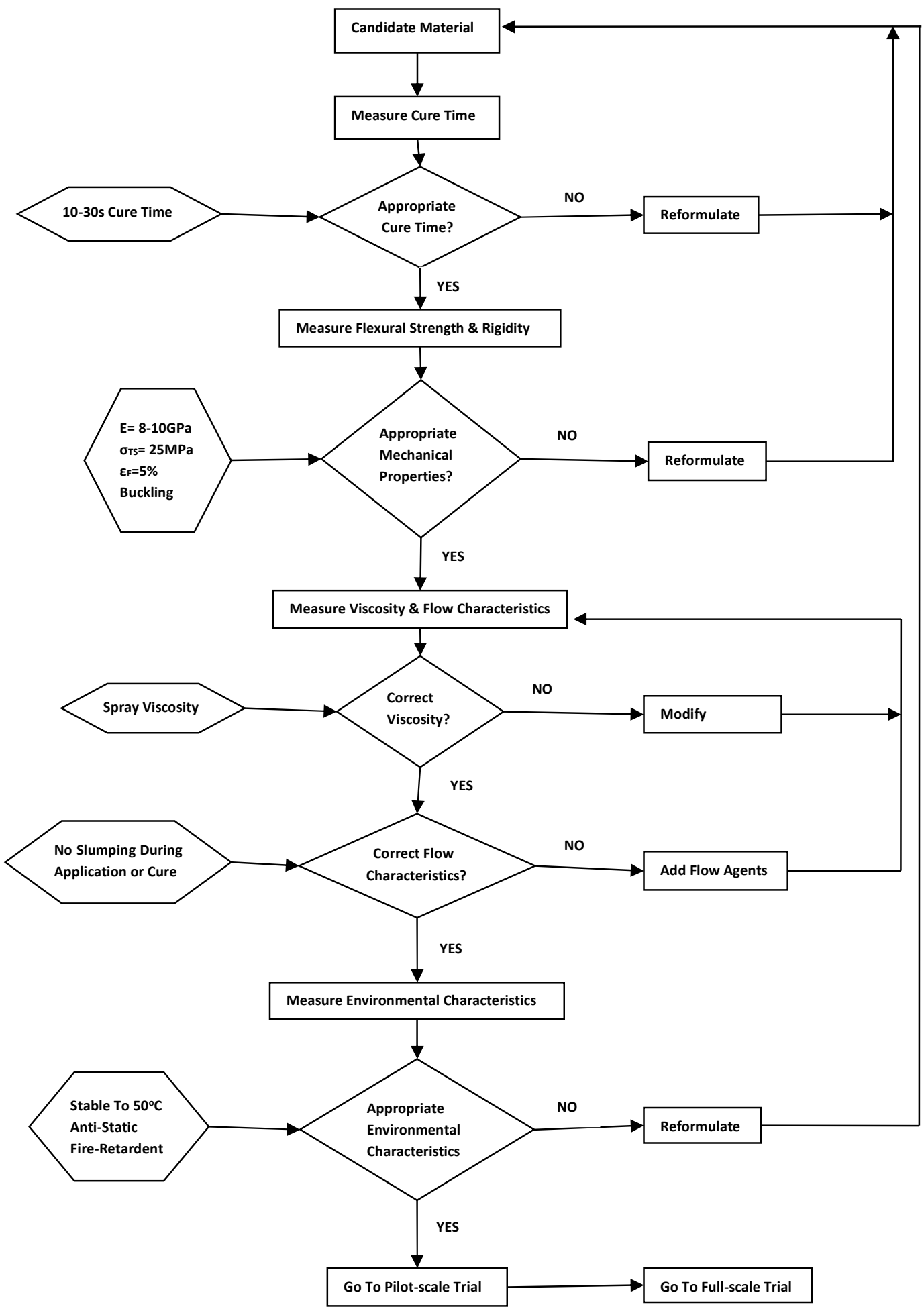

Fig. 1. Flowchart for selection of candidate materials. 


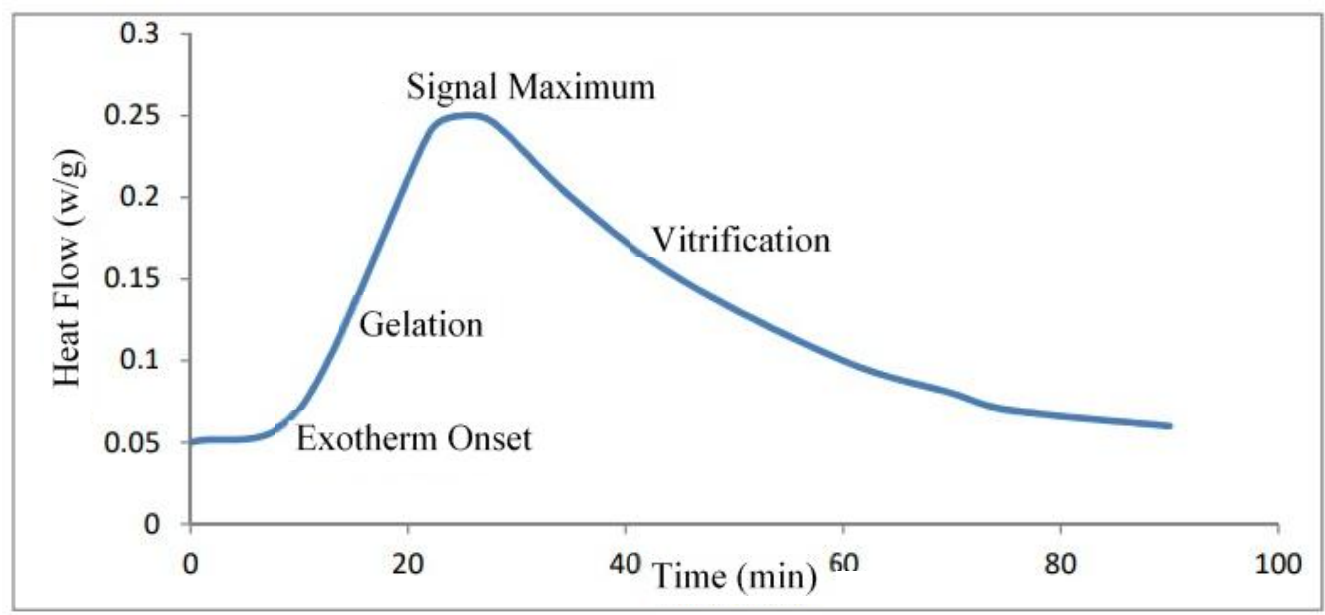

Fig. 2. Typical DSC measurement of crosslinking polymer cure.

\subsection{Flexural Properties}

There is a noticeable effect of the movement in the underground mine strata occurred by the development of flexural loads. Then the necessity of understanding the flexural powers originated from both of the mesh \& its polymeric replacements is essential. In this content, a 3point bend test is done by causing some tested specimens to undergo for measuring the flexural strength of those materials in the laboratory. For this test, a rectangular shape beam is selected to make it a bending material under some external loads. Then the strength of the flexure can be calculated from the known peak load in conformity with the following mathematical expression:

Where,

$$
\sigma_{F}=\frac{3 F}{2 b h^{2}}
$$

$$
\begin{array}{ll}
\sigma_{F} & =\text { flexural strength }(\mathrm{MPa}) \\
F & =\text { maximum load reached }(\mathrm{N}) \\
L & =\text { distance between supports }(\mathrm{mm}) \\
B & =\text { sample width }(\mathrm{mm}) \\
h & =\text { sample thickness }(\mathrm{mm})
\end{array}
$$

Again, it is also practicable to calculate the maximum deflection $(\delta)$ of the material after measuring a new parameter tensile modulus $(\mathrm{E})$ of that material before it goes to the condition. The equation is as follow,

$$
\delta=\frac{r L^{3}}{4 E h^{3}}
$$

Hence, a constant rate of deformation is considered at $2 \mathrm{~mm} / \mathrm{min}$ for interpreting the flexural behavior of the reinforcing polymers \& the result are demonstrated in Figure 3, in which it can be seen that none of the materials is in the region of the catastrophic fragile failure, but in a little extent, a successive loss of the strength can be observed that it is caused by the appearance of the reinforcing filler. From the Figure 3, it is observed that there are two types of formation of the materials. One formation has the strong flexibility with less strength, where other formation has the high fragility with low strength. Hence, a wide variety of flexural properties has been measured from the number of polymers \& then tested for displaying its range. From this test, a polymer with the best suitable properties is chosen for the desired modeling of the mesh. 


\subsection{Rheology}

The allowable range of viscosity is essentially defined \& controlled, so that the spray of the new material would be efficient. Here, not only are the introductions of the rheological properties important to spray the applied material without any type of slumping, but it is also better if an additional 'thixotropic agent' can be added with the formulation to reduce the slumping $\&$ the result is evaluated experimentally later. Therefore, a viscometry measurement can be helpful here to address the evaluation of both of these experiments.

\subsection{Environmental Characteristics}

Copied with the rheological properties, some environmental issues are also needed for controlling the toxicity, $\mathrm{pH}$ tolerance, temperature equilibrium $\&$ anti-static quality. Hence, the glass transition temperature is used to convert the cross-linked polymer to a state of low modulus rubbery solid from the high modulus glassy solid. For stabilizing the underground temperature under the existing condition, the transition temperature of the glassy polymer is in the recommended temperature exceeding about $50^{\circ} \mathrm{C}$.

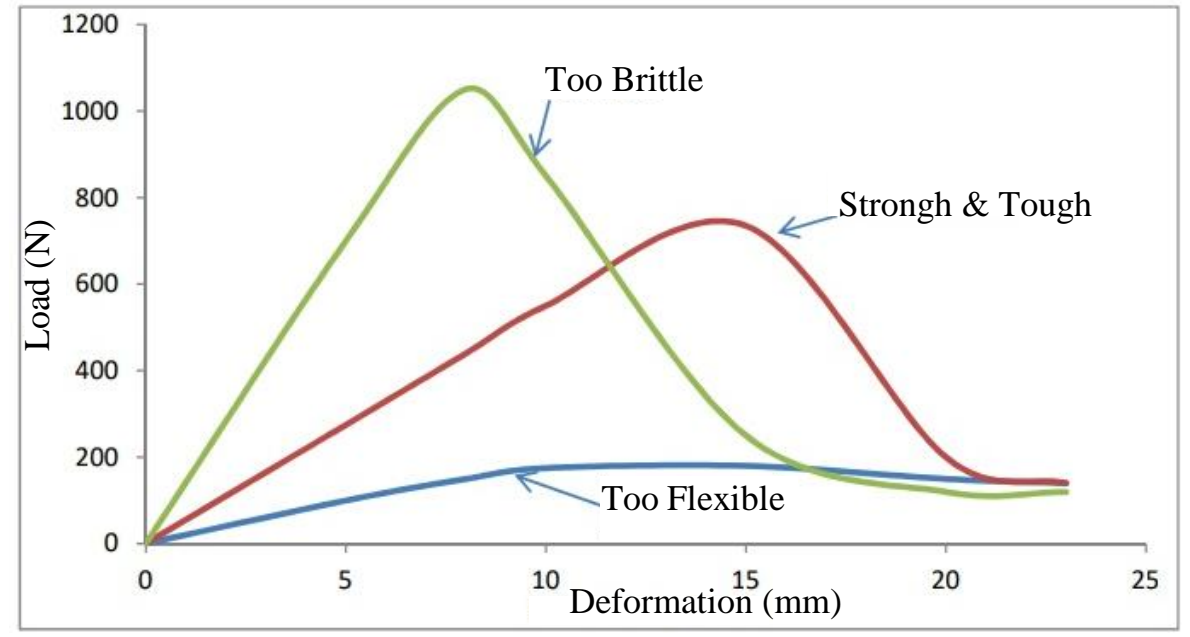

Fig. 3. Flexural tests on candidate reinforced polymers.

As observed above, it is found that water can cause a problem in the mines. The measured $\mathrm{pH}$ of mine water can be ranged from 2 to 10 so that the physical properties of the new used material must be freed from the attack of mine water's $\mathrm{pH}$ when it is associated with the wet coal. Therefore, some methods such as ASTM \& Mine safety methods include the explosive, toxic \& anti-static properties are implied in that context.

\section{Critical Governing Issues for Material Selection}

The results to date shown in figure 3 indicate that the selection of crosslinking polymers as the alternative replacement of steel mesh depends on the governing issues. From these points, some issues may be addressed, so that potential material is considered appropriated for the underground application. For marking the exact potential issues, a process \& product risk evaluation has been put in effect which is responsible for identifying the following critical issues:

- Dimension of toxicity \& degree of inflammatory at the time of application;

- Long life;

- Product quality control;

- Suitable mechanical properties of product; 
- Material should not be static \& not spread fire;

- Any type of unfavorable effect should not be in the coal plant during the clearing \& preparing times.

\subsection{Application Quality Control}

It is examined that a uniform ground condition does not exist everywhere in the mine. In some underground mines, there is a presence of uniformed thickness between the bolts \& their vicinity of the sections. It is also observed that there is no formation of any smooth surface when the fresh coal seam is cut. For these reason, the significance of quality control of the application has been increased gradually. Therefore, implication of the polymeric mesh as a replacement of the steel mesh should be done in persistent manner so that the replacement would be properly automated in the existing ground conditions.

\subsection{Health Issues}

There are some health related issues associated with the use of polymeric alternatives that can be observed in the underground mines in large amounts. These health issues are mainly related with the possibility of having emissions of any toxic or irritant gases during the installation or application of the material. In many cases, the cause of the emissions from these gases is the reaction between the volatile chemical products during the cure. In a confined space in the underground during the cure, these volatile products emit not only water, but also some toxic or explosive gases. Therefore, between the polymer \& cure chemistries, any type of condensation process should not occur. During the time of selecting raw materials for this application, a major investigation must be done to remove the possibility of using toxic \& irritant materials.

\subsection{Mechanical Properties}

The actual purpose of this work is to find out the best polymeric material as the best alternative of the steel mesh with the equivalent properties. Therefore, firstly it is necessary to determine the mechanical properties of steel mesh before the polymer-based alternatives for the convenience of the measurement. Here, steel mesh is produced from the low carbon containing steel wire that was welded in a square mesh pattern. Generally, the $4 \%$ volume of the mesh comes from steel by which it can provide a rough calculation of the tensile strength of the steel mesh towards the approach of the wire. Some measurements related with the tensile properties of the steel mesh \& wire, \& crosslinking polymers are given in Table 1 . If we neglect the effect of the strain failures, then the tensile strength properties of the steel meshes are similar with the crosslinking polymers. Actually, a geotechnical representation of the underground steel mesh leading with a realistic \& robust system should be designed to ascertain the endurable mechanical properties of the polymeric alternatives for the steel mesh.

Table 1. Typical tensile properties of steel mesh, wire \& reinforced crosslinking polymer

\begin{tabular}{cccc}
\hline PROPERTY & $\begin{array}{c}\text { LOW CARBON } \\
\text { STEEL WIRE }\end{array}$ & $\begin{array}{c}\text { LOW CARBON } \\
\text { STEEL MESH }\end{array}$ & $\begin{array}{c}\text { CROSSLINKING } \\
\text { POLYMER } \\
\text { 30-50\% FIBRE }\end{array}$ \\
\hline Yield Strength (MPa) & $500-600$ & $20-24$ & $25-55$ \\
\hline Young's Modulus (GPa) & $205-215$ & 8 & $10-17$ \\
\hline Tensile Strength (MPa) & $500-600$ & $20-24$ & $30-70$ \\
\hline Failure Strain (\%) & $4-6$ & $4-6$ & $1-2$ \\
\hline
\end{tabular}

The other important issue is to observe the measurement of adhesion promptness between the coal strata \& polymeric material. The next plan should be to employ the material, which can achieve the initial cure in a while to go through bolting properly \& make the material stable 
dimensionally. Sometimes, this type of characteristic of wet or uncured material is also effectible $\&$ has been seen as a potential skin mechanism of an uncured or reinforced material. Hence, a test of measuring this adhesion strength is made by taking samples from various types of rock \& coal stratum under several conditions.

\subsection{Longevity}

Some leading issues associated inherently with the polymer replacement mechanisms that can ensue at any time of the process are the degradation of both thermal \& strength properties, weathering, environmental stress breaking etc. These thermal \& processing degradations usually happen as a cause of occur, the open exposure to the outside temperature during the manufacturing \& synthesizing processes of the finished polymer in application services. Generally, this case does not occur all-time, because the temperature in mine environment anyhow is considered stable. Thermal degradation is almost an uncommon case for the mine area.

Weathering is also a type of degradation occurred by exposing to the weather. When a polymer based material is exposed to weather, then a ray of ultraviolet light combined with the atmospheric oxygen can cause that polymer to be a shoot of the weathering. This type of weathering process is known as 'Photo-oxidation' (Rabek 1995). Without the appearance of the atmospheric oxygen on the exposure, no solar UV light can affect the polymer. Therefore, like the previous thermal degradation, weathering is also an uncommon issue in the underground mines.

Environmental stress combines tensile stress, involve the forming \& developing of cracks \& finally accelerate in reaching the ultimate point of the rock failure. As the dimension of the ground movement is changed gradually in the mine roadways, the stress originated from the skin confinement is also changing constantly. However, some polymers are highly capable to restrain this stress $\&$ some are less capable. Accelerated examination of any probable nominated material should be performed to determine the longevity of the polymer during the service.

\subsection{Anti-Static and Fire Retardancy}

Polymers are made from some constituents that are generally insulated for electricity. When an air flows through the material surface, then the friction is created on the surface to stack the static electricity resulted from that airflow. That accumulated electricity has been build up gradually to form sparking outflow which can lead up to an underground fire. In this case, polymers are unfortunately less efficient than the steel mesh, because steel mesh is susceptible enough to conduct this static electricity in safe way. To make the polymer based materials efficient for this criterion, several additives are included with those polymer materials. Generally, coal dust is considered as the best anti-static additives for alleviating this problem (Grob \& Minder 1999). Because, the coal dust is made from graphitic carbon that is conductive electrically, but before that, the amount of the coal dust needed for using as additive should be examined in laboratory by fulfilling the legislation \& regulation.

The other major noticeable thing of using polymer based material is its remarkable fire retardant tendency. Because, polymers are generally combustible with a spontaneous flammability where steel meshes are not combustible \& flammable. The polymers are made from the incorporation of a number of monomers which are produced from the fire retardant chemical elements or additives. This type of tendency of the polymer alternatives are reduced by two means. First one is, adding of the fire-suppressants into the polymer to yield some non-combustible gases such as $\mathrm{CO}_{2}$ etc. which can lead to the reduction of flammability (Biswas et al. 2007). The second one is the adding of the intumescent additives swell up \& the carbonization of the polymer alternatives by eliminating the set up of the flame from the surface (Ma et al. 2007). Therefore, these two types of ways are suitable for removing the above mentioned problems related to the use of the polymer based alternatives. 


\section{Conclusion}

A polymer based material as an alternative to the steel mesh is employed to offer many expedients than using mesh. Therefore, all the issues related with this desired polymeric material should be addressed afore to evaluate its capacity. An estimation found from a reliable research has stated that a number of developed characteristics of a sustainable polymer based material as replacement of the steel mesh can excel all the issues narrated above during the advancement of the underground coal mine roadways. Not only that, but also the problems related with the downstream processing in the coal mine roadways can be solved by using a polymeric alternative. However, some researches should be going on for reducing the firing \& explosion tendency of the polymer alternative chemical elements along with the reduction of toxicity \& environmental hazards during the time of both in application \& cure. This can be expected that if the differences of the roles of steel \& polymer meshes are understood properly, then further consideration of development can be held in the underground coalmine roadways.

\section{Acknowledgements}

The author gratefully acknowledges the help of the one of my course coordinators. He had helped me with providing some information on this topic to write this paper.

\section{References}

[1] Espley-Boudreau, S.J. (1999). Thin spray-on liner support and implementation in the hardrock mining industry. M.A.Sc. thesis, Laurentian University, School of Engineering, Sudbury, Ontario, Canada.

[2] Potvin, Y., Stacey, T.R.., Hadjigeorgiou, J. and Yilmaz, H. (2004). Thin spray-on liners a quick reference guide. Surface Support in Mining, edited by Y. Potvin, T.R.. Stacey and J. Hadjigeorgiou, p7.

[3] Chang, M.-C. and Chen, S.-A. (1987). Kinetics and mechanism of urethane reactions: phenylisocyanate-alcohol systems. J. Polymer Sci.: Part A: Polymer Chemistry, 25, 2543.

[4] Martin, J.S., Laza, J.M., Morras, M.L., Rodriguez, M. and Leon, L.M. (2000). Study of the curing process of a vinyl ester resin by means of TSR and DMTA. Polymer 41, 4203.

[5] Cook, W.D., Lau, M., Mehrabi, M., Dean, K. and Zipper, M. (2001). Control of gel time and exotherm behavior during cure of unsaturated polyester resins. Polymer Int., 50, 129.

[6] Rabek, J.F. (1995). Polymer Photodegradation. Chapman \& Hall, London.

[7] Grob, M.C. and Minder, E. (1999). Permanent antistatic additives: new developments. Plastics, Additives \& Compounding, July 1999, 20.

[8] Biswas, B., Kandola, B.K., Horrocks, A.R. and Price, D. (2007). A quantitative study of carbon monoxide and carbon dioxide evolution during thermal degradation of flameretarded epoxy resins. Poly. Deg. Stab., 92,765.

[9] Ma, H., Tong, L., Fang, Z., Jin, Y. and Lu, F., (2007). A novel intumescent flame retardant: synthesis and application in ABS copolymer. Poly. Deg. Stab., 92, 720. 